\title{
Compatibility Estimation of Oligobutadienediol with Simple Oligoetherpolyols for Molded Polyurethane Compositions Development
}

\author{
IVAN. A. NOVAKOV ${ }^{1}$, VASILIY. P. MEDVEDEV ${ }^{1}$, MARAT. A. VANIEV ${ }^{1}$, GEORGIY.V. \\ MEDVEDEV ${ }^{1}$, SAULE A. SAKIBAYEVA², ANASTASSIYA. Y. KOVALEVA ${ }^{2 *}$ \\ ${ }^{1}$ Volgograd State Technical University, Russian Federation, 400005, \\ Volgograd, Lenin av., 28, Kazakhstan. \\ ${ }^{2}$ M. Auezov South Kazakhstan State University, Kazakhstan, Shymkent, \\ Tauke khan av., 5, Kazakhstan. \\ *Corresponding author E-mail: anastasiya2301@ mail.ru \\ http://dx.doi.org/10.13005/ojc/330518
}

(Received: July 15 2017; Accepted: August 20, 2017)

\begin{abstract}
This article includes the results of compatibility of oligomeric hydroxyl-containing butadienerubber with oligoetherpolyols on the basis of propylene oxide (Laprols). The compatibility limits of liquid hydroxyl-containing butadiene rubber of Krasol LBH-3000V brand with polypropyleneglycols of Laprol brand with different molecular weights were determined.
\end{abstract}

Keywords: Rubber, Oligomer, Oligoetherpolyol, Oligoetherdiol, Polyurethane, Functional group, Chain branching agent.

\section{INTRODUCTION}

Molded polyurethane systems based on oligoetherpolyols and oligobutadienediols are widely used in various items production, produced on free molding technology. Polyether polyurethane are characterized by a rather high level of strength characteristics and relatively low cost, what explains their wide use in practice. Polyether polyols based on propylene oxide under the general name of Laprols were the most widely used for the preparation of polyurethanes. However, it should be noted that they have a low hydrolytic stability. It limits their application at high humidity, under the influence of minus temperatures and significant temperature differences ${ }^{1}$.

Polyurethanes based on oligodienediols have exceptionally high hydrolytic stability, but they are much inferior to polyetherurethanes for their strength properties. In addition, oligodienediols are more expensive and therefore less available. 
Nowadays for these purposes, oligobutadiene isoprenediol of the brand PDI-1 and oligo but a dienediol of the Sartomer production of the brand Krasol $\mathrm{LBH}^{2}$ are used ${ }^{2}$.

In this regard, the actual task is to find new binders or modify existing ones, in order to increase hydrolytic stability, while maintaining high strength characteristics, reducing the cost of molding compositions and materials based on them.

One of the possible ways to solve this problem is to combine the oligoether and oligodienediols to produce polyurethanes that meet the above requirements ${ }^{3}$.

\section{MATERIALS AND METHODS}

Krasol LBH-3000V (MM $\approx 2800-3200 ; \rho=$ 0,9 ; hydroxyl number - 1,4\%) - oligomeric rubber containing butadienehydroxil. Krasol LBH-3000V is a low molecular polybutadiene with tail hydroxyl groups.

Structural formula of this substance is:

$$
\begin{array}{cc}
\mathrm{CH}_{3} & \mathrm{CH}_{3} \\
\mathrm{HO}-\mathrm{I} & \mathrm{C} H-\mathrm{CH}_{2}-\left[\mathrm{CH}_{2}-\mathrm{CH}=\mathrm{CH}-\mathrm{CH}_{2}\right]_{n}-\mathrm{CH}_{2}-\mathrm{C} \mathrm{CH}-\mathrm{OH}
\end{array}
$$

At normal temperature Krasol LBN-3000V is a viscous, colorless or yellowish liquid, immiscible with neither water, nor with alcohols.

Laprol 3003 (Laprol 373, Laprol 2102, Laprol 4503, Laprol 5003) is a polyether of molecular weight, obtained by alkaline polymerization of propylene oxide and glycerol. Laprol is used as a polyol stocks for the production of rigid polyurethane foams used in the manufacture of heat-insulating and structural products.

Researched oligomeric products are characterized with different polarities. The molecular chains of the Krasol LBH 3000 rubber have a hydrocarbon butadiene nature, at the ends of the chains are located hydroxyl groups, the content of which is $1.1-1.4$ percent. Such a chemical structure of macromolecules determines the low polarity of the rubber. In order to take into account the degree of compatibility and predict the possibility of the emergence of thermodynamically stable systems at the heteropolar polymers mixing, a compatibility evaluation is necessary. The evaluation of compatibility can be carried out both theoretically and experimentally. In this article, the compatibility evaluation was carried out by several methods.

One of the simplest ways to quantify the ability of polymers to coincide is to determine the relationships of solubility parameters ${ }^{4}$, which are characterized with the specific cohesive energy or the energy of intermolecular interaction.

For polymers, Small ${ }^{4}$ proposed to calculate the solubility parameter on the basis of interaction energies conditional values of the various atoms groups of the polymer chain consisting of the main link according to the equation:

$$
\delta=\rho * \sum \mathbf{F}^{*} \mathbf{M}^{-1}
$$

where $\rho$ - polymer's density, $F$ - the energy of interaction of a certain group of atoms, $\mathrm{M}$ - molecular weight of a repeating unit.

Theoretical determination of the compatibility of Krasol/Laprol polymers pair can be made by calculating the Gibbs energy of a binary system by mixing, using the Flory-Huggins equation ${ }^{5}$ :

$$
\Delta \mathrm{G}=\frac{R T V}{\mathrm{Vc}}\left[\frac{\varphi_{\mathrm{a}}}{\mathrm{r}_{\mathrm{a}}} \ln \varphi_{a}+\frac{\varphi_{\mathrm{b}}}{\mathrm{r}_{\mathrm{b}}} \ln \varphi_{b}+\chi_{a b} \varphi_{a} \varphi_{b}\right]
$$

where $\mathrm{V}$ - total volume of mixture, $\mathrm{V}_{\mathrm{c}}-$ segment volume; $r_{a}, r_{b}$ - number of segments of polymers, $r_{i}=V_{i} / V_{c}$ (where $V_{i}$ - molar volume of component); $\varphi_{\mathrm{a}}, \varphi_{\mathrm{b}}$ - volume fractions of polymers; $\chi_{\mathrm{aB}}-$ thermodynamic interaction parameter.

Since the researched compatibilized polymers are liquids, it seems reasonable to use a visual method for the experimental determination of compatibility by the appearance of the interface of an exfoliating system with a certain ratio of components. This is a simple and quick method for compatibility assessment, known as the Dobri and Bowyer-Covenwick method. For this purpose two polymers are poured together, mixed thoroughly, and the mixture is poured into the tube. The first 3 days tubes are in the oven at a temperature of $100^{\circ} \mathrm{C}$.

The rest of the time the tubes are at room temperature $\left(15-27^{\circ} \mathrm{C}\right)$. If the solution remains 
transparent, the polymers are considered as compatible; if the solution becomes cloudy or delaminated into two layers, each containing mainly one of the polymers, the polymers are considered as incompatible. The delamination of polymers mixture is observed at determined for each pair of polymer ratios. Observations were carried out for 60 days.

The mutual solubility of the polymers is determined by measuring the optical density of the two polymers mixture. If the polymers are mutually soluble, the mixture is transparent, if they are insoluble, then the particles of the second phase are formed, which are the centers of scattered light, which leads to a change in the optical density, the addition of small amounts of the second polymer does not cause changes in the optical density. When the amount of the second polymer exceeds its solubility, the mixture becomes cloudy, the optical density increases, indicating the formation of the second phase.

The optical density of polymer mixtures was determined on a spectrophotometer SF 56 with a software product that allows scanning the spectra in the wavelength region 190-1100 $\mathrm{nm}$ with $1 \mathrm{~nm}$ sampling increment. The thickness of the polymers mixture layer placed in the measuring cuvette is $1 \mathrm{~mm}$.

Samples are prepared in the laboratory using a mixer LM-1 with an anchor-type stirring device with a rotation speed equals $252 \mathrm{rpm}$.

At the end of the mixing process, the mixture coats the molds pre-coated with the release agent. The depth of the mold is 5 millimeters. The rate of samples curing is judged by the increase in hardness over time.

\section{RESULTS AND DISCUSSION}

In results of the research it was determined that Laprols and liquid rubber are characterized by a complicated molecular structure and chemical composition of macromolecules. To evaluate the solubility parameters, their experimental determination or calculation by known methods is necessary. One of the calculated methods is Small's method, which takes into account the energy of interaction of all the links of the groups of atoms entering into the structure of the macromolecule. The calculation of solubility parameters is given in Table 1.

The calculated values of solubility parameters allow to suppose that the most compatible will be the oligomers pairs of Krasol LBH 3000 and Laprol 3003 having the smallest difference in solubility parameters. The rubber $\mathrm{LBH}$ 3000 and Laprol 373 are less compatible, the solubility parameters differ in a greater degree.

However, this method allows to carry out only preliminary compatibility analysis, in order to better assess the compatibility, including to determine the quantitative ratio of mutually soluble binary systems, this method must be supplemented with additional techniques.

Gibbs energy $\Delta \mathrm{G}$ concentration dependence for researched binary systems at different temperatures are given on the picture. 1 and picture. 2. Value calculation of $\Delta \mathrm{G}$ shows that in different binary pairs rubber-Laprol the different conditions for achieving thermodynamic stability are required. In the rubber-Laprol 373 system, maximum stability is achieved only with insignificant additions of Laprol (no more than 10-20\%) of the polyester. In the system rubber-Laprol 5003 the maximum stability is maintained at higher concentrations (up to 70:30 ratio) of Laprol 5003 in the mixture. In the rubber-Laprol 2102 system, the share of Laprol could be increased to $40-50 \%$. For the remaining pairs with Laprol 3003 and 4503, the share of Laprols in the mixture can reach from 40 to $60 \%$. It should be noted that deviating ratio of the components from the above does not imply the immediate exceeding of the system from thermodynamic equilibrium and loss of stability, since the $\Delta G$ values remain negative.

With an increase in temperature, an increase in compatibility is observed while maintaining a range of concentrations with maximum stability. 
The data obtained in the aggregate with the compatibility estimation on the value of solubility parameters also indicate the highest compatibility of Laprols 3003 and 4503 with rubber. However, this method does not allow us to determine the limiting relationships at which the mixture will delaminate.

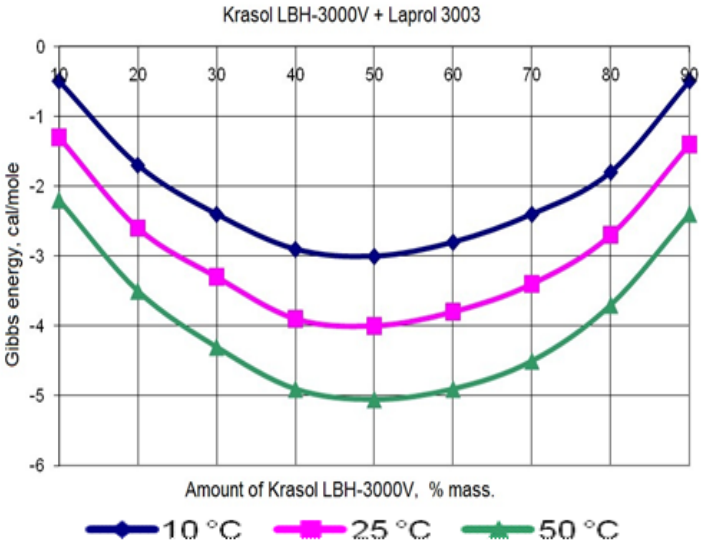

Fig.1. Dependence of free Gibbs energy of mixing from Krasol LBH-3000 V contentin the system for the Krasol LBH-3000V/Laprol 3003 system at different temperatures.

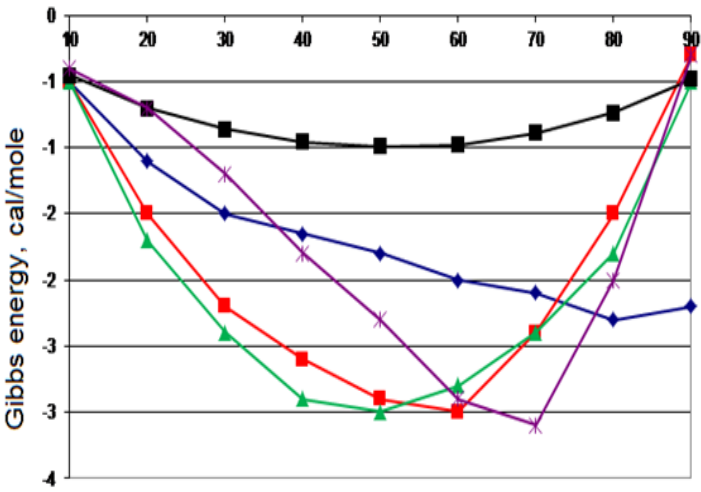

Amount of Krasol LBH - 3000V. \% mass.

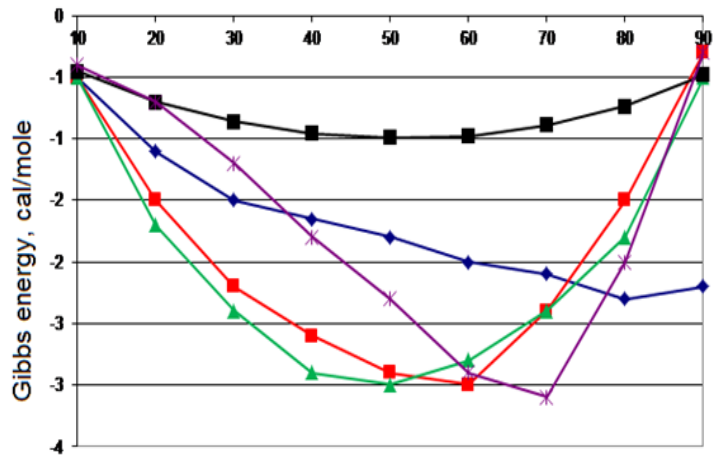

Amount of Krasol LBH - 3000V. \% mass. 


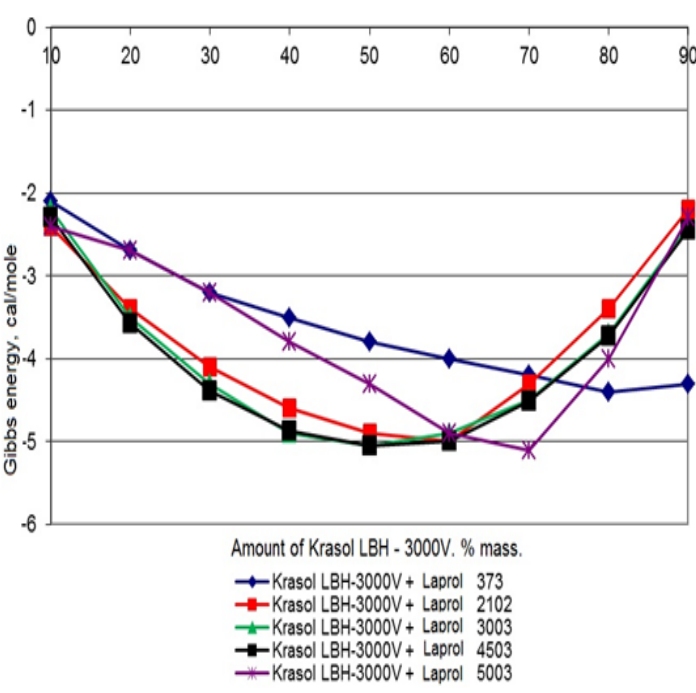

Fig.2. Dependence of free Gibbs energy of mixing from rubber content in the mixture for the system Krasol LBH-3000V/Laprol 3003 at different temperatures $\left(\alpha-10^{\circ} \mathrm{C} ; \partial-25^{\circ} \mathrm{C} ; \alpha-50^{\circ} \mathrm{C}\right)$

During the mixing boundedly compatible liquids in quantitative ratios exceeding the solubility limit of one liquid in another, spontaneous delamination of the mixture into two layers or turbidity occurs as a result of microbundling. In the first case, a clearly visible interface is formed. This phenomenon is used to determine the limits of compatibility of liquids, as the most accessible and accurate method. To determine the limiting quantitative compatibility ratios of rubber-Laprol pairs, researched mixtures of various ratios were held at $100{ }^{\circ} \mathrm{C}$ in glass tube until the interface appeared. After 3-7 days, the system is delaminated for incompatible mixtures.

The results of the analysis are almost the same with combining estimation on the basis two methods, with more exact determination of specific interconnection. It was invented the most compatible compliant combination rubber-Laprol 3003 and less compliant compatible oligomers rubber-Laprol 373. However, for some combinations, inspite of the fact of phases separation, it is also impossible to determine the limit of compatibility. In particular, for rubber-Laprol
2102 and 4503 mixtures, a rather wide range of ratios is observed, the turbidity of mixtures of which indicates incomplete compatibility at concentrations of Laprols in a mixture much lower than the limiting one. This can lead to an erroneous determination of the separation limit.

An analysis of the results of the compatibility assessment of binary mixtures carried out by various methods (project MES RK 1529/Г $\varphi 4$ ) made it possible to establish that the oligopropylene glycol Laprol 3003, which is the most compatible among the pairs under research, is the most suitable for combining with butadiene oligomeric rubbers. Stability of the mixtures is preserved when 40- $50 \%$ of Laprol is added to the rubber, spontaneous separation occurs only when the proportion of Laprol in the mixture is brought up to $53 \%$. Laprol 2102 and 4503 have sufficiently high compatibility of rubber mixtures. The rubber systems with Laprol 5003 and 373 are the least compatible.

\section{CONCLUSION}

The compatibility research of butadiene low molecular hydroxyl-containing rubber with polypropylene glycols was carried out, which allowed to determine the limits of mutual solubility and choose the most compatible polyester component for the modification of oligomeric compositions. It was found that it is the most expedient to use oligopropylene glycol, Laprol 3003 to combine with butadiene oligomeric rubber, which is characterized by the greatest compatibility among the pairs under study. Based on the obtained results, in the future research in this field the most compatible systems will be used to determine the influence of rubber-Laprol ratio and other formula factors on the technical properties of polyurethanes.

\section{ACKNOWLEDGEMENT}

The research is implemented in the framework of the project $\mathcal{N}_{0}, 4.3230 .2017 / 4.6$.

\section{REFERENCES}

1. MedvedevV.P. Polyurethane elastomers on the basis of oligobutadienediol Krasol LBH
3000 // Adhesives. Sealants. Technologies, 2012, 11, 17. 
2. Medvedev V.P., Chapurkin V.V., Ukrainskaya S.I. Structure and properties of polyurethane elastomers on the properties of oligodienediol with 1,2-structure of double bonds // Rubber and resin, 2012, 1,2.

3. V. Medvedev, M. Vaniyev, S. Sakibayeva, A. 5. Kovaleva. Oligomers solidification depending on the nature, molecular mass, type and reactional group containing// Oriental Journal of Chemistry, 2017, 1, 269273.

4. Shvarc A.G. Rubbers combination with synthetic tars. Chemistry, 1972, 224.

Andreev V.N., Petrov T.P. Catalysts of oligodieneisocyanates polymerization // HMC, 1970, 9, $654-657$. 